\title{
A fatal case of encephalitis
}

\begin{abstract}
Encephalitis is a serious neurological condition; delayed diagnosis of encephalitis can cause significant mortality and morbidity. Even timely treatment may not be enough to save the patient. In this article we report a case of herpes encephalitis which was treated and discharged from the intensive care unit with cure, but readmitted with status epilepticus with a relapse of encephalitis. Herpes relapse was dismissed by PCR and although the diagnostic workup was inconclusive the patient was diagnosed as autoimmune encephalitis. The patient's seizures couldn't be controlled for 175 days and all treatment attempts failed to reverse the clinical situation. The patient was lost during the intensive care treatment.
\end{abstract}

Keywords: encephalitis, herpes simplex, autoimmune, status epilepticus
Volume 2 Issue I - 2015

\section{Ipek Edipoglu, Seval Urkmez, Tughan Utku, Mustafa Akker, Yalim Dikmen \\ Department of Intensive Care, Cerrahpasa Medical School, Turkey}

Correspondence: Ipek Edipoglu, Cerrahpasa Medical School, Departmentof Intensive Care, 34098 Fatih, Istanbul,Turkey, Fax +90212 414 3588, Email ipekeipoglu@gmail.com

Received: December II, 2014 | Published: February 19, 2015
Abbreviations:VGKC, voltage-gated potassium channel; NMDAR, N-methyl, D-aspartate receptor; LGI 1, leucin-rich gliomainactivated-1; CASPR 2, contact in-associated protein-2; SE, status epilepticus; WBC, white blood count; ALT, alanine transaminases; AST, aspartate transaminases; HSV-1: herpes simplex virus; PCR, polymerase chain reaction; EEG, electro encephalogram; CT, computed tomography; CJD, creutzfeldt-jakob disease; ECT, electro convulsive therapy; CPT2, carnitine palmitoyl transferase II

\section{Introduction}

Encephalitis is a serious neurological condition, which must be diagnosed and treated immediately. Delayed diagnosis of encephalitis can cause significant mortality and morbidity. ${ }^{1,2}$ It may not always be possible to identify the underlying etiology. Acute encephalitis has wide variety of clinical manifestations. Patients may have mild symptoms like diminished consciousness or they may have mortal clinical course complicating with coma, seizures and even multiorgan failure. ${ }^{3}$ Infections are common causes of encephalitis. Viruses constitute a major proportion of infectious encephalitis. Herpes simplex $(90 \% \mathrm{HSV}-1)$ is the leading pathogen of viral encephalitis. Its incidence for United States is 2000 cases per year. ${ }^{4}$ But recently non-infectious etiologies are increasingly acknowledged by the intensive care practitioners. ${ }^{2}$

The emphasis and awareness about autoimmune etiology for encephalitis has extended significantly in updated literature. ${ }^{5}$ To our knowledge, auto antibodies against surface neuronal proteins are likely to be responsible for autoimmune encephalitis syndromes. Often these antibodies target against the voltage-gated potassium channel (VGKC) and N-methyl, D-aspartate receptor (NMDAR), and recent studies showed that VGKC is not a single target for antibodies but it is the "VGKC-complex, which they aim. VGKC-complex is found tightly within the mammalian brain membranes and made up of leucine-rich glioma-inactivated-1 (LGI1), contact in-associated protein-2 (CASPR2) and contactin-2. ${ }^{6}$ In this report we present a mortal case of acute autoimmune encephalitis, which was preceded by herpes encephalitis (Figure 1), manifested with refractory status epilepticus (SE) unresponsive to any given therapy. Although the knowledge is intense about acute encephalitis in literature, this young adult with encephalitis was not consistent with any other subtypes.

\section{Case report}

A 17 years old male was presented with syncope and seizures following tooth extraction. He attended to a different clinic with the syncope and seizure symptoms on the following day. As his clinical progression went worse, he was transferred to our hospital for advanced diagnosis. He went into status epilepticus (SE) three days after the extraction, on the day we administered him to the ICU. His medical history was unremarkable. His fever was $36.5^{\circ} \mathrm{C}$ with arterial tension $125 / 75 \mathrm{mmHg}$, heart rate $84 / \mathrm{min}$, Kernig-Brundzki sign negative. Lumbar puncture results revealed insignificant cell count, cerebrospinal fluid (CSF) glucose: $72 \mathrm{mg} / \mathrm{dl}$ and protein: $35 \mathrm{gr} / \mathrm{dl}$. His cranial MRI revealed findings concordant with left temporal lobe encephalopathy. His blood tests were, CRP: $101 \mathrm{mg} / \mathrm{L}$, white blood count (WBC): $11000 \mathrm{~mm}^{3}$, neutrophils: \%82, lymphocyte: \%10, platelet: 269000, Hgb: $13.5 \mathrm{~g} / \mathrm{dl}$, Urea: $34 \mathrm{mg} / \mathrm{dl}$, creatine: $0.9 \mathrm{mg} / \mathrm{dl}$, alanine transaminases (ALT): $34 \mathrm{IU} / \mathrm{L}$, aspartate transaminases (AST): 32IU/L, ESR: $15 \mathrm{~mm} / \mathrm{h}$, blood glocose: $83 \mathrm{mg} / \mathrm{dl}$, anti-HIV: negative. His electrolyte levels were normal. Herpes simplex virus (HSV-1) was detected with polymerase chain reaction (PCR) technique in his CSF. Initial diagnosis was herpes simplex virus (HSV-1) and acyclovir treatment had been started at the admission along with ceftriaxone and metronidazole. The seizures were controlled with anticonvulsive and the patient was awake and cooperative. As the general condition improved the patient was discharged from the intensive care unit to the infectious diseases ward on the fourth day of his admission.

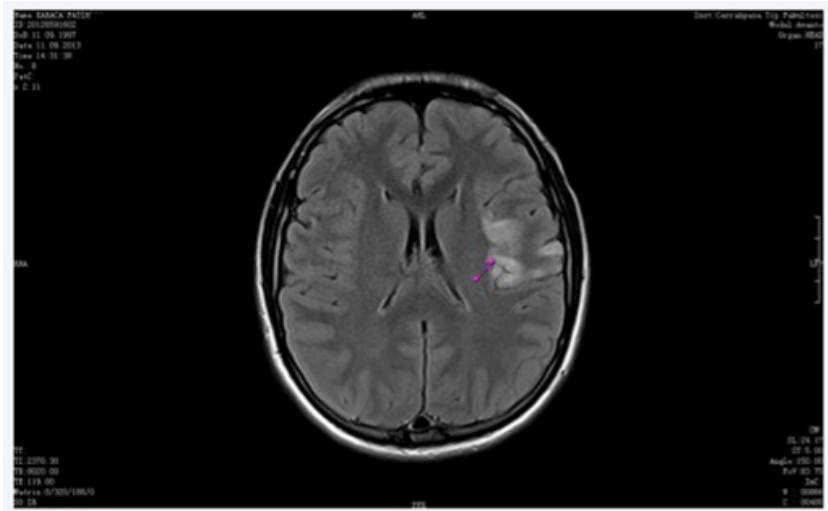

Figure I First magnetic resonance imaging consistent with herpes encephalitis.

Four days after discharge the patient was readmitted to the ICU with status epilepticus. He was incubated and sedated with propofol to control seizures. His electroencephalogram (EEG) revealed seizure activity concordant with SE. Levotiracetam and valproic acid therapy was initiated. On the fourth intensive care day, cerebral angiography showed lesions associated with mitochondrial myopathy, encephalopathy, lactic acidosis, and stroke (MELAS) syndrome. Although at first lesions detected in the angiography was in favor 
of ischemia, we could not detect any lesions in the communicant arteries and this finding directed us to MELAS syndrome for the diagnosis. Cranial computed tomography (CT) was normal on the 9th day. Cranial and neck contrast enhanced MRI showed diffusion restriction in the anterior and inferior of left temporal lobe on the 11th day. Radiologists revealed that the lesion in the medial lobe was newly formed and suggested us to consider infection or autoimmune encephalitis for the etiology in the foreground. And we shifted the diagnosis from MELAS syndrome to autoimmune encephalitis. Diffusion restriction was newly formed in left medial temporal cingulate gyrus in his diffusion MRI on 12th day. The CSF analysis with PCR for herpes infection was negative (Figure $2 \& 3$ ).
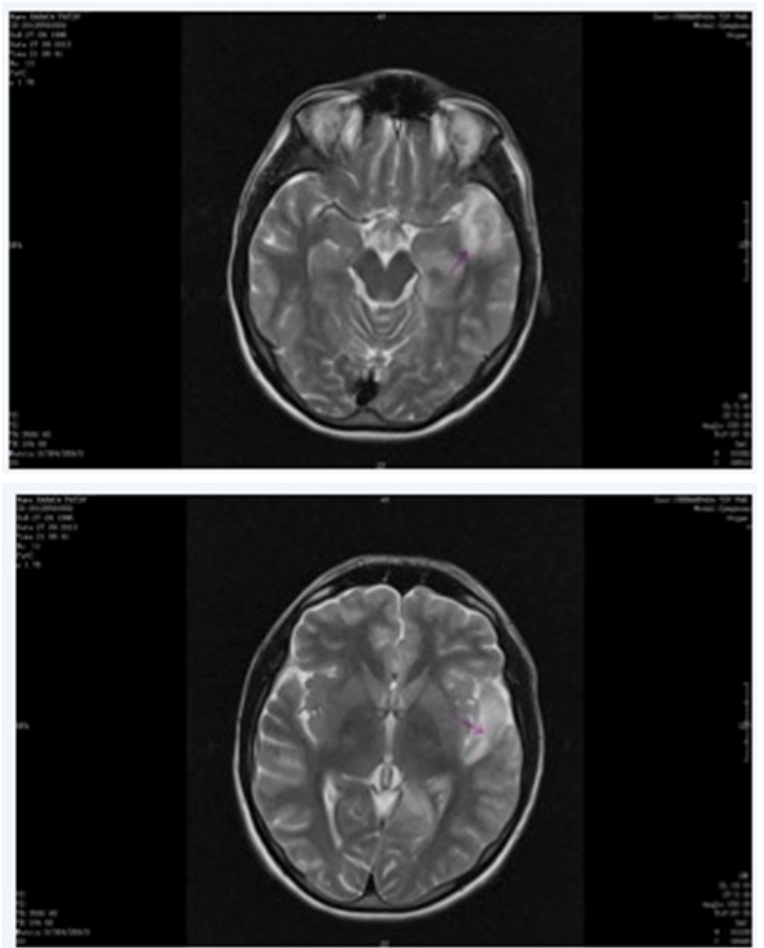

Figure 283 $\mathrm{MRI}$ in the second admission, increased signal intensity in anterior and posterior aspects of left temporal lobe. Lesions are progressive with new ones spread in brain tissue, infection or autoimmune encephalitis should be considered.

Levotiracetam and valproic acid therapy was not successful in controlling seizure activity, that's why; we initiated midazolam and propofol infusion therapy with intermittent diazepam when necessary. Pulse steroid (methylprednisolone) and IVIG therapy (3 times) was also initiated for autoimmune encephalopathy treatment. Blood tests were made for ANA, ANCA, anti-cardiolipin IgM-IgG, anti phospholipid IgG-IgM to eliminate central nervous system vasculitis. And their results were all negative. Thyroid function tests were made for the diagnosis of Hashimoto disease but these were negative also. On the 43th day of ICU stay with no apparent progress, Plasmapheresis for 5 days and cyclophosphamide therapy once a month (for 3months) was initiated, when pulse steroid therapy was inefficient. During the stay a ketogenic diet was initiated to help to stop seizures, which was proved to be ineffective, and was stopped on the 45th day. A percutaneous dilatational tracheostomy was performed on the 65th day; it was delayed for too long with the anticipation of controlling the seizures and extubating the patient. On the 82th day thiopental infusion was started as there had been no other choice to stop the seizure activity. The control CT revealed hydrocephaly secondary to diffuse cerebral atrophy (Figure 4).

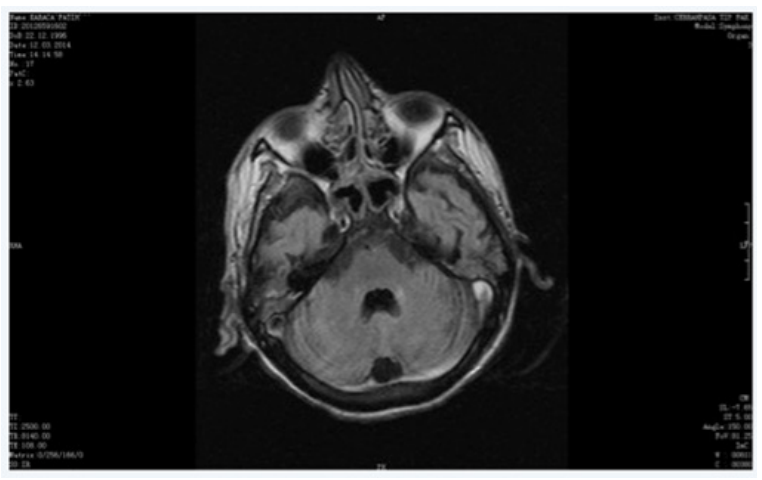

Figure 4 Last MRI showing diffuse cerebral atrophy.

On the 113th day a new cranial MRI showed that active neuropathological condition persisted, but interestingly the affected lobe of the patient was shifted to the contra lateral side. In his previous MRI's his left hemisphere were affected but this MRI revealed that the disease was affecting right hemisphere. Our patient was discussed in the epilepsy surgery council and the council decided not to operate as he would not benefit from the surgery. Lactic acid and 3-OH-isovaleric acid levels were not significant in his urine. Cerebrospinal fluid and serum rubella antibodies were negative. CSF tests for detecting prion or SSPE were all insignificant. Protein 14.3.3 for Creutzfeldt-Jakob disease (CJD), TAU protein and protein S were also negative. His B12 levels were $184.9 \mathrm{U}$ and necessary B12 replacement was made. Urine organic acid and amino acid analysis were negative for the diagnosis of urea cycle defects and organic acidemia. Ammonium and lactate levels were normal for monoclonal gammopathy of undetermined significance (MGUS) diagnosis. Blood and urine metal levels were also normal to eliminate heavy metal intoxication. In order to restrain seizure activity, electro convulsive therapy (ECT) was performed. Para neoplastic encephalopathy was eliminated with negative antineuronal antibody levels and negative abdominal CT. USG for the diagnosis of testicular tumor was also negative.

Anakinra (IL-1 receptor antagonist) therapy was given to the patient after pulse steroid, intravenous immunoglobulin and Plasmapheresis therapies all failed to treat refractory status epilepticus. But we could not manage to suppress seizure activity. The patient died 175 days after his readmission to our ICU.

\section{Discussion}

We report a case of fatal encephalitis with severe progressive clinical course refractory status epilepticus and diffusion restriction in left medial temporal cingulate gyrus. We considered the case as autoimmune encephalitis as we could not be able to detect any other cause. All diagnostic tests we have performed were negative, even the autoimmune test panel was negative. This is the reason why we considered our diagnosis as autoimmune encephalitis from an unknown subtype. LoPinto-Khoury and Sperling ${ }^{7}$ suggest considering status epilepticus as autoimmune if it is refractory to anticonvulsant therapy and a specific factor cannot be determined for the etiology. Then empiric immuno modulatory treatment should be considered. In our case we managed status epilepticus of our patient similar to LoPinto-Khoury's suggestions but not able to suppress seizure activity.

Ando et al. ${ }^{3}$ recently reported two cases of acute encephalopathy with distinctive clinical manifestations. Both of their patients had SE and marked brain edema with bilateral thalamic lesions on the MRI. 
One of their patients had thermo labile variant of carnitine palmitoyl transferase II (CPT2) gene, which has a possible contribution to acute encephalitis. And a genetic variation might have affected our patient as well.

Sasaki et al. ${ }^{8}$ reported 41 cases of pediatric autoimmune encephalitis in a retrospective study and concluded that children with acute encephalitis concordant with refractory SE have a poorer neurological outcome and longer intensive care stay. For nearly half of the autoimmune encephalitic children an etiological agent cannot be detected. Our case was very similar with this cohort, showing very poor outcome and a long hospital stay without exact known etiology. Although there is not a conclusive guideline for the treatment of autoimmune encephalitis, authors generally suggest using steroids as the first line therapy, followed by IVIG and Plasma-pheresis, when steroids are inadequate or contraindicated. Cyclophosphamide is also suggested. ${ }^{5,9}$ We followed the therapy suggestions for our patient and treated as advised but all therapies were unsuccessful.

IL-1receptor antagonist drugs (anakinra) are widely studied in clinical trials for their potential effects on autoimmune and inflammatory diseases. These studies directed the investigators to look for any impending treatment about IL-1 antagonists in epilepsies associated with pro inflammatory conditions. ${ }^{10}$ In animal studies anakinra reduces seizures, inhibits cortical generalization from the hippocampus and decreases SE frequency. ${ }^{11-14}$ After all possible therapies failed, we decided to use anakinra with the light of promising clinical trials, and its known effects over immune and inflammatory processes. Informed consent was taken from the family to use the drug. But even with IL-1 receptor antagonist we could not manage to suppress the seizure activity.

\section{Conclusion}

We report a patient with acute encephalitis and concomitant refractory status epilepticus. Definitely, as we achieved our diagnosis with the exclusion of diseases; it is not possible to reach a definitive conclusion for our case. Its resistance to all possible therapies and poor prognosis directed us to consider the encephalitis as autoimmune etiology. However we still have uncertainties about the possibility of herpes encephalitis whose PCR tests may sometimes become negative during follow up period. A sour case did not show any similarities with the published cases, an unknown subtype of autoimmune encephalitis or a genetic disorder may be the reason that hinders our diagnosis

\section{Acknowledgments}

None.

\section{Funding details}

None.

\section{Conflicts of interest}

Authors declare that there is no conflict of interest.

\section{References}

1. Glaser CA, Honarmand S, Anderson LJ, et al. Beyond Viruses:Clinical Profiles and Etiologies Associated with Encephalitis. Clin Infectious Dis. 2006;43(12):1565-1577.

2. Wingfield $\mathrm{T}, \mathrm{McHugh} \mathrm{C}$, Vas $\mathrm{A}$, et al. Autoimmune encephalitis:a case series and comprehensive review of the literature. QJM. 2011;104(11):921-931.

3. Ando N, Okumura A, Kobayashi S, et al. Fulminant Encephalopathy with Marked Brain Edema and Bilateral Thalamic Lesions. Neuropediatrics. 2013;45(4):256-260.

4. Kennedy PG. Viral encephalitis:causes, differential diagnosis, and management. J Neurol Neurosurg Psychiatry. 2004;75Suppl 1:i10-i15.

5. Ramanathan S, Mohammad SS, Brilot F, et al. Autoimmune encephalitis:Recent updates and emerging challenges. $J$ Clin Neurosci. 2014;21(5):722-730.

6. Irani SR, Vincent A. The expanding spectrum of clinically-distinctive, immunotherapy-responsive autoimmune encephalopathies. Arq Neuropsiquiatr. 2012;70(4):300-304.

7. LoPinto-Khoury C, Sperling MR. Autoimmune Status Epilepticus. Curr Treat Options Neurol. 2013;15(5):545-556.

8. Sasaki J, Chegondi M, Raszynski A, et al. Outcome of children with acute encephalitis and refractory status epilepticus. J Child Neurol. 2014;29(12):1638-1644.

9. Vitaliani R, Zoccarato M, Vianello M, et al. Clinical, immunological and therapeutic aspects of autoimmune encephalitis. Recent Pat CNS Drug Discov. 2008;3(1):16-22.

10. Vezzani A, Balosso S, Maroso M, et al. ICE/caspase 1 inhibitors and $\mathrm{IL}-1$ beta receptor antagonists as potential therapeutics in epilepsy. Curr Opin Investig Drugs. 2010;11(1):43-50.

11. Vezzani A, Moneta D, Conti M, et al. Powerful anticonvulsant action of IL-1 receptor antagonist on intra cerebral injection and astrocytic over expression in mice. Proc Natl Acad Sci USA. 2000;97(21):11534-11539.

12. Vezzani A, Moneta D, Richichi C, et al. Functional role of inflammatory cytokines and anti inflammatory molecules in seizures and epileptogenesis. Epilepsia. 2002;43(Suppl 5):30-35.

13. Marchi N, Fan Q, Ghosh C, et al. Antagonism of peripheral inflammation reduces the severity of status epilepticus. Neurobiol Dis. 2009;33(2):171-181.

14. Vezzani A, Bartfai T, Bianchi M, et al. Therapeutic potential of new ant inflammatory drugs. Epilepsia. 2011;52(Suppl 8):67-69. 